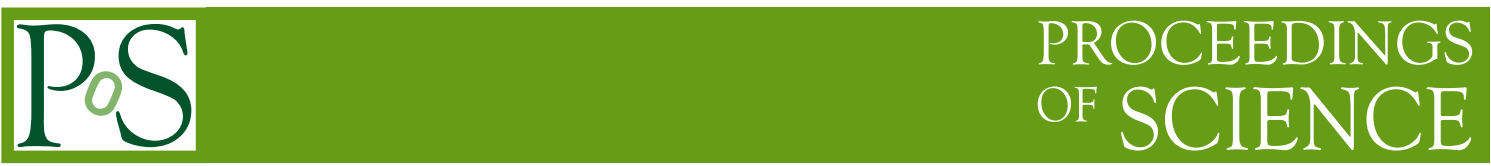

\title{
When jets MET SUSY: ATLAS searches for squarks and gluinos
}

\author{
Speaker: Aaron Paul O'Neill on behalf of the ATLAS collaboration ${ }^{a, *}$ \\ ${ }^{a}$ University of Oxford, \\ Oxford, England \\ E-mail: aaron.paul.o'neill@cern.ch, \\ ATLAS-publication-committee-chair@cern.ch
}

\begin{abstract}
In many supersymmetric scenarios, heavy Beyond Standard Model particles would decay to multiple massive Standard Model bosons or top quarks. The subsequent decays of these Standard Model particles into leptons and/or jets may then occur with significant branching ratios and can populate the events recorded by the ATLAS detector. This talk presents ATLAS searches for coloured superparticles decaying to jets and missing transverse momentum, which utilise cutting-edge object and event reconstruction to seek a buried signal in the full Run 2 LHC dataset. Combining these methods with powerful statistical analyses allows novel constraints to be applied to natural and unnatural SUSY, as well as shedding light on the existence of Dark Matter and other novel particles. Constraints on squarks and gluinos in final states populated by one or more leptons are also presented.
\end{abstract}

40th International Conference on High Energy physics - ICHEP2020

July 28 - August 6, 2020

Prague, Czech Republic (virtual meeting)

\footnotetext{
${ }^{*}$ Speaker
} 


\section{Introduction}

The ATLAS experiment [1] recorded a phenomenal amount of data from 2015 to 2018, known as Run 2; a total integrated luminosity of $139 \mathrm{fb}^{-1}$. The huge increase in recorded data can be seen in Figure 1. Could new physics in the form of an extension to the standard model of particle physics (SM), in particular supersymmetry (SUSY), be hiding in this enormous dataset?

This note describes the latest improvements in jet reconstruction as well as improvements in the calculation of missing transverse momentum $\left(E_{\mathrm{T}}^{\text {miss }}\right)$ and its significance $\left(\mathcal{S}\left(E_{\mathrm{T}}^{\mathrm{miss}}\right)\right)$. Then how these improvements impacted the results from strongly produced SUSY (squark $(\tilde{q})$ and gluino $(\tilde{g})$ production) searches using these techniques.

\section{Jet Reconstruction}

Jets are extremely important objects in the ATLAS detector, therefore it is critical that they are well understood and calibrated correctly. In the simplest terms, jets are collimated sprays of particles measured by their energy deposits in the detector. The energy scale and corresponding resolution for jets was measured using ATLAS data with an integrated luminosity between $36-81 \mathrm{fb}^{-1}$ (2015 - 2017) and Monte Carlo simulation [3].

There are several methods to build jets from detector inputs and it is becoming increasingly common to use particle flow (PFlow) jets [4]. These objects are designed to combine track

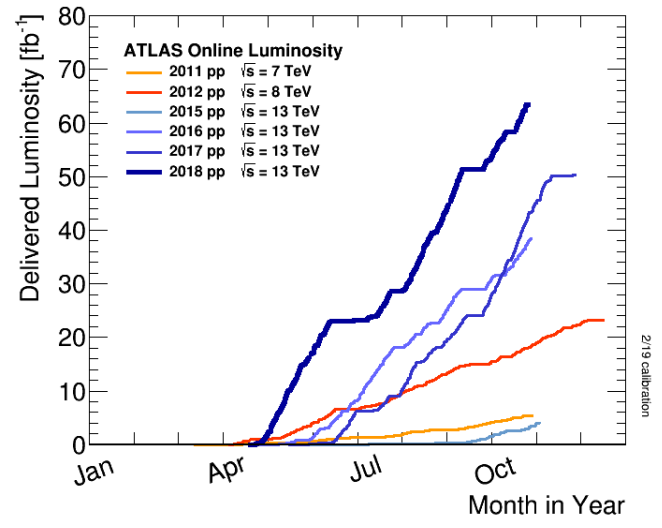

Figure 1: Cumulative luminosity versus day delivered to ATLAS during stable beams and for high energy $p-p$ collisions [2]. information from the inner detector with the calorimeter clusters. PFlow jets offer increased performance over electromagnetic topocluster jets (EMTopo) [5], which use only topological clusters of calorimeter cells as inputs, due to superior track momentum resolution at low momentum. The momentum resolution of the tracker [4],

$$
\sigma\left(\frac{1}{p_{\mathrm{T}}}\right) \cdot p_{\mathrm{T}}=0.036 \% \cdot p_{\mathrm{T}} \oplus 1.3 \%
$$

shows the performance is best at lower transverse momentum $\left(p_{\mathrm{T}}\right)$ and degrades as the $p_{\mathrm{T}}$ becomes large. However, as the energy of an object increases the calorimeter is increasingly used to form the jets as it has improving performance at higher energies, which is demonstrated by the resolution as a function of energy $(E)$,

$$
\frac{\sigma(E)}{E}=\frac{50 \%}{\sqrt{E}} \oplus 3.4 \oplus \frac{1 \%}{E} .
$$

The energy from a track (or tracks) associated with calorimeter cells is subtracted. This removes the energy deposited by all charged particles in the calorimeter and the jet reconstruction is performed 
on a collections of 'particle flow objects'; one set representing the charged objects and the other being the neutral particle flow objects made up of the remaining calorimeter deposits after track energy subtraction.

At high enough energies the tracker is no longer used and thus the performance of PFlow is comparable to EMTopo jets. Utilising the tracker for PFlow jets has several other advantages over EMTopo jets including; improved angular resolution coming from the superior positional resolution of the tracking detector and reduction of extra energy energy entering jets from simultaneous collisions in the proton bunches (pileup).
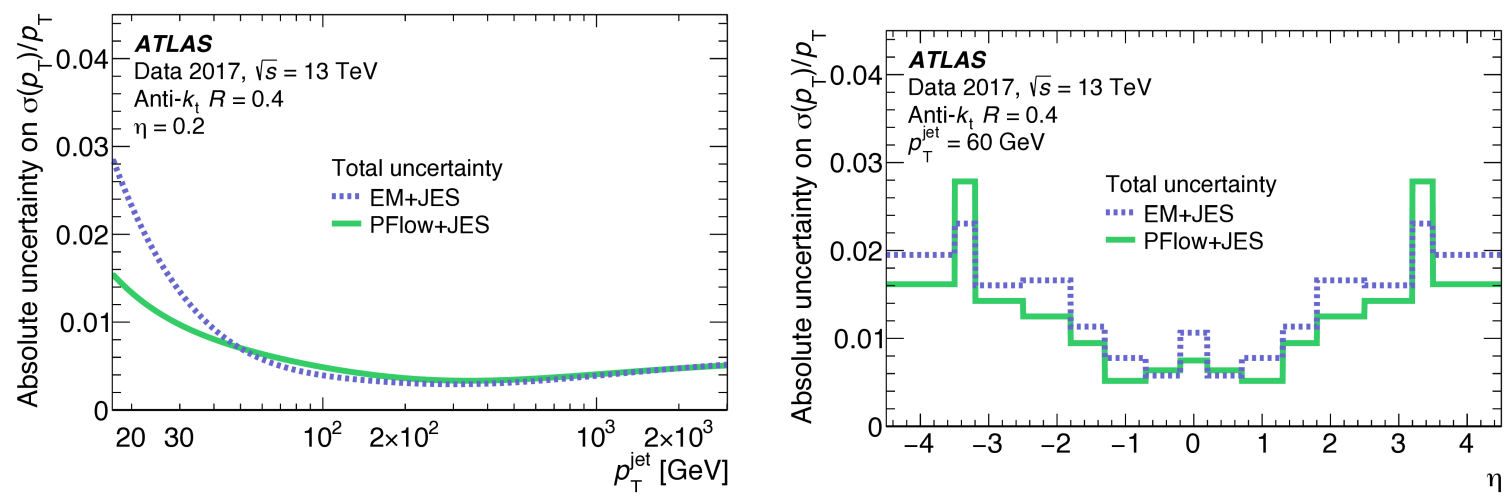

Figure 2: The absolute uncertainty for PFlow (with jet energy scaling (JES)) jets, in green, and EMTopo + JES, in blue, as a function of jet $p_{\mathrm{T}}$ (left) and the jet pseudo-rapidity or $\eta$ (right). In the $p_{\mathrm{T}}^{\text {jet }}$ distribution all of the jets considered have $\eta=0.2$ and in the $\eta$ the jets have $p_{\mathrm{T}}=60 \mathrm{GeV}$ [3].

All of these improvements result not only in better jet reconstruction and a reduction in pileup but also a suppression of the absolute uncertainty as seen in Figure 2. The transverse momentum of the jets $p_{\mathrm{T}}^{\text {jet }}$ (Figure $2\left(\right.$ left) ) demonstrates a reduction in absolute uncertainty at low $p_{\mathrm{T}}^{\text {jet }}$ for PFlow and then as the momentum increases the performance becomes comparable to EMTopo jets. The right hand distribution of pseudo-rapidity $(\eta)$ shows that the uncertainty in $\eta$ is reduced across most of the total range for PFlow jet reconstruction.

\section{Missing Transverse Momentum $\left(E_{\mathrm{T}}^{\mathrm{miss}}\right)$ and Its Significance $\left(\mathcal{S}\left(E_{\mathrm{T}}^{\mathrm{miss}}\right)\right)$}

Missing transverse momentum $\left(E_{\mathrm{T}}^{\mathrm{miss}}\right)$ is a vital quantity in ATLAS as it is a measure of the transverse momentum carried away by particles invisible to the detector. In the SM there is only one particle type that will carry $p_{\mathrm{T}}$ invisible to the detector; the neutrinos. Aside from this a great many new physics models, including SUSY, require the existence of invisible particles.

The latest definition of $E_{\mathrm{T}}^{\text {miss }}$ is a negative vector sum of all the reconstructed and calibrated physics objects in the detector plus another component called the soft term. The physics objects are added to the sum in a particular order (Equation 3): muons, electrons, photons, hadronic $\tau$, jets and finally the soft term. This order is to prevent double counting of energy deposits in the detector, all of the objects except the soft term are collectively known as the hard term $\left(\boldsymbol{p}_{\mathrm{T}}^{\text {hard }}\right)$. 
The soft term $\left(\boldsymbol{p}_{\mathrm{T}}^{\text {soft }}\right)$ is made up of all remaining detector signals not associated to any of the physics objects. There are two ways of constructing the soft term; the first is to use tracks from the inner detector (ID) to form the track soft term (TST) and the second is to use the left over calorimeter deposits, topo-clusters, to form the calorimeter soft term (CST). For the rest of this note the track soft term will be used as it demonstrates stronger pileup resistance. With all of these components we have the object-based missing transverse momentum,

$$
\boldsymbol{E}_{\mathrm{T}}^{\mathrm{miss}}=-\left(\sum_{i \in \text { muons }} \boldsymbol{p}_{\mathrm{T}}^{i}+\sum_{i \in \text { electrons }} \boldsymbol{p}_{\mathrm{T}}^{i}+\sum_{i \in \text { photons }} \boldsymbol{p}_{\mathrm{T}}^{i}+\sum_{i \in \text { hadronic } \tau} \boldsymbol{p}_{\mathrm{T}}^{i}+\sum_{i \in \text { jets }} \boldsymbol{p}_{\mathrm{T}}^{i}+\sum_{i \in \mathrm{SoftTerm}} \boldsymbol{p}_{\mathrm{T}}^{i}\right)
$$

Once the $E_{\mathrm{T}}^{\text {miss }}$ has been calculated how do we know if it comes from a real invisible particle carrying momentum away from the experiment undetected and not from resolution effects? This is where the definition of a significance becomes useful, the missing transverse momentum significance $\mathcal{S}\left(E_{\mathrm{T}}^{\text {miss }}\right)$ is a measure of how confident one is that the measured $E_{\mathrm{T}}^{\text {miss }}$ is real. Object-based $E_{\mathrm{T}}^{\text {miss }}$ significance is an event-by-event evaluation of of the $p$-value that the observed $E_{\mathrm{T}}^{\text {miss }}\left(E_{\mathrm{T}}^{\text {miss,obs }}\right)$ is consistent with the null hypothesis that there is no real missing transverse momentum, $E_{\mathrm{T}}^{\text {miss,true }}=0$. In the frame of the $E_{\mathrm{T}}^{\text {miss }}$ vector the significance can be written in the simple form

$$
\mathcal{S}^{2}=\frac{\left|\boldsymbol{E}_{\mathrm{T}}^{\mathrm{miss}}\right|^{2}}{\sigma_{\mathrm{L}}^{2}\left(1-\rho_{\mathrm{LT}}^{2}\right)}
$$

where $\sigma_{\mathrm{L}}^{2}$ is the longitudinal $(\mathrm{L})$ variance and $\rho_{\mathrm{LT}}^{2}$ is the correlation between the longitudinal and transverse $(\mathrm{T})$ variance. In the frame of $\boldsymbol{E}_{\mathrm{T}}^{\text {miss}}$; longitudinal means parallel to the vector and transverse is perpendicular to the same vector. This definition encodes the true definition of a significance, the variable is in the numerator and the description of its variance is in the denominator.
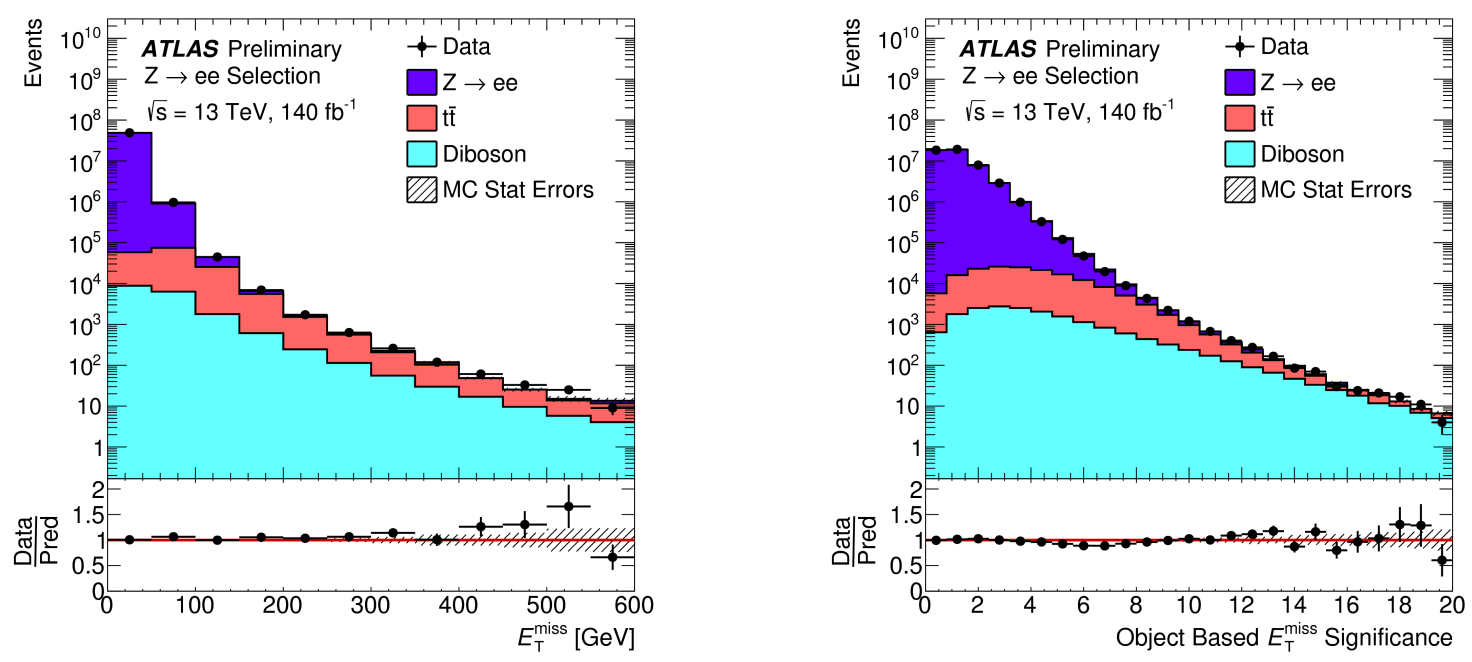

Figure 3: The $E_{\mathrm{T}}^{\text {miss }}$ (left), in $\mathrm{GeV}$, and object-based $E_{\mathrm{T}}^{\text {miss }}$ significance (right) distributions for the $Z \rightarrow e e$ event selection. Both figures show the results using the entire run 2 dataset. Each of the processes contributing are represented by different MC simulations; $Z \rightarrow e e$ in purple, $t \bar{t}$ in red and diboson processes in turquoise $[8]$. 
The modelling of $E_{\mathrm{T}}^{\text {miss }}$ and $\mathcal{S}\left(E_{\mathrm{T}}^{\text {miss }}\right)$ can be tested by comparing Monte Carlo (MC) to data for a particular selection. A standard candle test is to use events containing a $Z$ boson decaying to two electrons $(Z \rightarrow e e)$. This final state should have no real missing transverse momentum and so be a good test of our calculation as there should be a large peak at low values of $E_{\mathrm{T}}^{\text {miss }}$ and the higher values of $E_{\mathrm{T}}^{\mathrm{miss}}$ should come from processes with neutrinos present. This is exactly what Figure 3 shows and also that the prediction by MC agrees excellently with the data. The right hand plot in Figure 3 displays the $E_{\mathrm{T}}^{\text {miss }}$ significance for the same event selection and as one would expect the $Z \rightarrow e e \mathrm{MC}$ (with no real missing transverse momentum) dominates the low significance values corresponding to $E_{\mathrm{T}}^{\text {miss }}$ coming from mismeasurement and resolution effects while the high values are coming from processes that have a neutrino present ( $t \bar{t}$ and diboson).

\section{Track Soft Term Uncertainty}

The systematics for each of the physics objects entering the hard term calculation are calculated separately and details of this will not be given here. Instead the track soft term systematics calculation will be described. The systematics for the TST are determined using projections relative to the transverse momentum vector of the hard term $\left(\boldsymbol{p}_{\mathrm{T}}^{\text {hard }}\right)$ and the the missing transverse momentum $\operatorname{vector}\left(\boldsymbol{E}_{\mathrm{T}}^{\mathrm{miss}}\right)$.

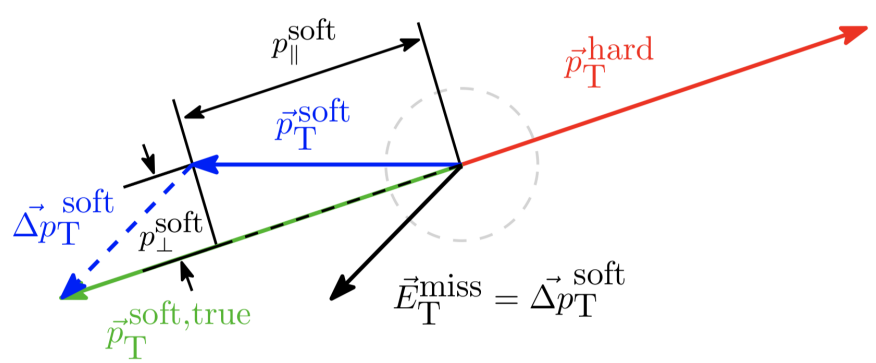

Figure 4: Schematic representation of the projection of the track soft term with respect to $\boldsymbol{p}_{\mathrm{T}}^{\text {hard }}$. These projections are used in the calculation of the TST systematics [6].

In a $Z \rightarrow e e$ event there should be no real missing transverse momentum and in an ideal world the momentum of the hard term is balanced by the momentum of the soft term, or $\boldsymbol{p}_{\mathrm{T}}^{\text {hard }}=-\boldsymbol{p}_{\mathrm{T}}^{\text {soft }}$, as seen in Figure 4. In the diagram this ideal case is represented by the green $\boldsymbol{p}_{\mathrm{T}}^{\text {soft,true }}$ vector but in reality the measured soft term will not balance perfectly and different projections of $\boldsymbol{p}_{\mathrm{T}}^{\text {soft }}$ along $p_{\mathrm{T}}^{\text {hard }}$ in data and MC can be used to study the modelling of the soft term.

There are three components used in the TST systematic and these projections can be seen in Figure 4. The first is the parallel scale $\left(\Delta_{L}\right)$ and this is the mean value of the parallel projection of $\boldsymbol{p}_{\mathrm{T}}^{\text {hard }}$ along $\boldsymbol{p}_{\mathrm{T}}^{\text {soft }}$; labelled as $\boldsymbol{p}_{\|}^{\text {soft }}$, the second term named the parallel resolution, $\sigma_{\|}$, is the RMS of the aforementioned parallel projection $\left(\boldsymbol{p}_{\|}^{\text {soft }}\right)$. The third component, which is related to the second, is the transverse resolution and this is the RMS of the transverse (perpendicular) component of $\boldsymbol{p}_{\mathrm{T}}^{\text {soft }}$ with respect to $\boldsymbol{p}_{\mathrm{T}}^{\text {hard }}$. This momentum component is labelled as $\boldsymbol{p}_{\perp}^{\text {soft }}$ and the resolution is denoted as $\sigma_{\perp}$ [6]. Figure 5 shows that particle flow jets offer an improvement in the largest component of the track soft term uncertainty, the parallel resolution. 

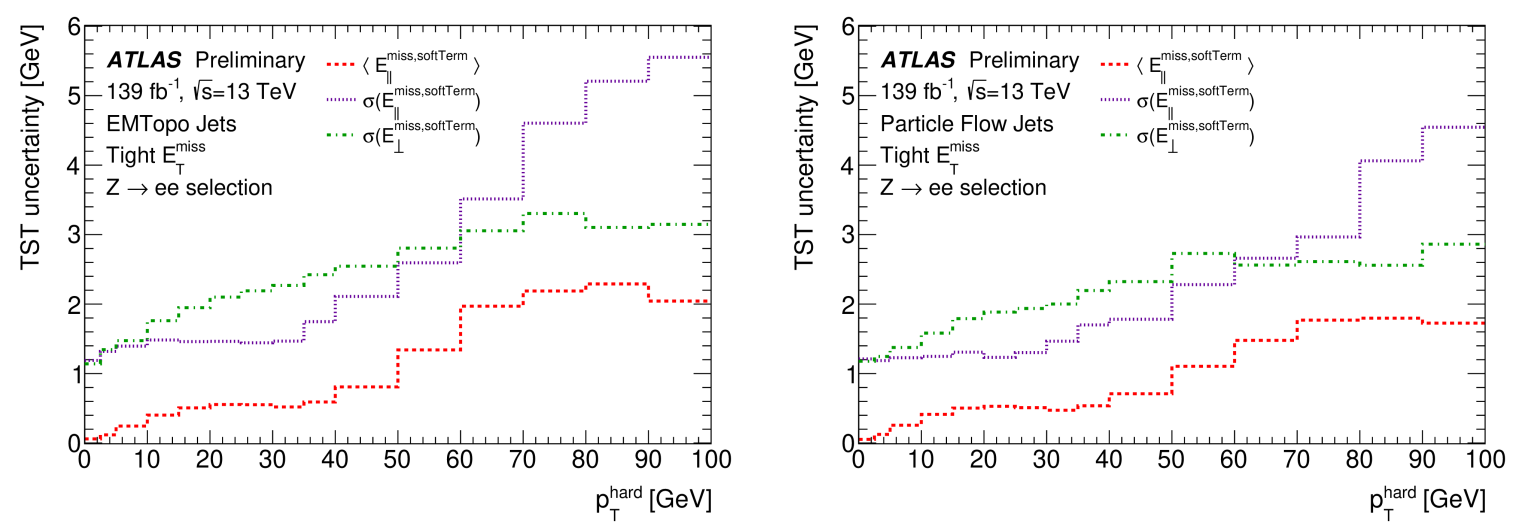

Figure 5: Each of the components of the track soft term uncertainties are shown for the two widely used jet types in ATLAS, both of these figures show the $Z \rightarrow e e$ event selection. The left hand plot details these components for EMTopo jets and the right shows the same for particle flow jets [9].

How do all of these performance improvements impact ATLAS analyses and in particular searches for physics beyond the standard model? The next two sections will describe two analyses that have availed of some or all of these advancements in detector performance.

\section{SUSY $t \bar{t}(2 L)+E_{\mathrm{T}}^{\text {miss }}$}

A search for pair production of top squarks $(\tilde{t})$ or dark matter (DM) particles $(\chi)$, coming from a spin-0 mediator particle $(\phi / a)$, that subsequently form events containing two jets, two opposite signed leptons (these can be electrons or muons) and missing transverse momentum. The analysis employed the new object-based $E_{\mathrm{T}}^{\text {miss }}$ significance described above and used the entire Run 2 ATLAS data set of proton-proton collisions at $\sqrt{s}=13 \mathrm{TeV}$ corresponding to an integrated luminosity of $139 \mathrm{fb}^{-1}[10]$.

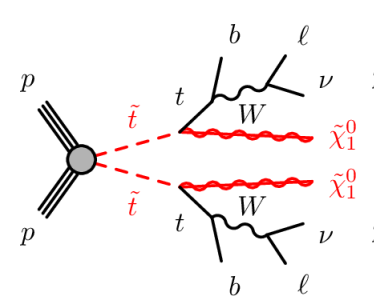

(a)

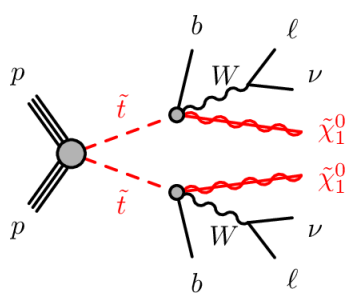

(b)

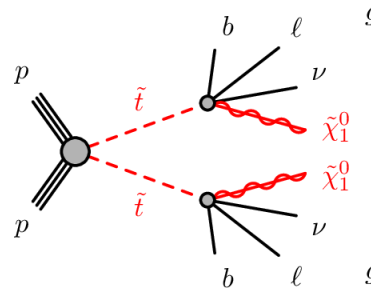

(c)

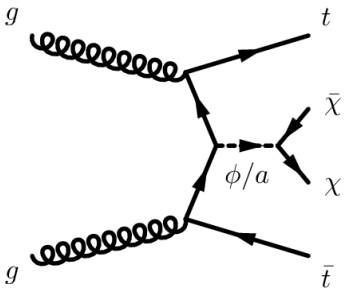

(d)

Figure 6: The four signal models targeted by the analysis. The first three are the supersymmetry models: (a) two-body decay shows the $\tilde{t}$ decaying directly to the top quark, which, in turn decays semi-leptonically to the bottom quark $\left(\tilde{t} \rightarrow t \tilde{\chi}_{1}^{0}\right)$, (b) three-body decay does not go via the standard model top quark, instead the $\tilde{t}$ decays directly to a bottom quark plus a $W$ boson $\left(\tilde{t} \rightarrow b W \tilde{\chi}_{1}^{0}\right)$, (c) the four-body channel removes the $W$ and the top squark goes straight to the bottom quark, a lepton and its neutrino $\left(\tilde{t} \rightarrow b l v \tilde{\chi}_{1}^{0}\right)$. The final diagram, (d), depicts the dark matter model, a spin- 0 mediator is able to decay to a pair of DM particles and this will happen in conjuction with the production of a pair of top quarks ( $p p \rightarrow \chi \bar{\chi} t \bar{t}$ ) [10].

Figure 6 shows the three supersymmetric signal models sought by the analysis as well as the DM model. It is worth noting that the DM model bares a striking resemblance to the two body final 
state from the squark decay and so is included in the two-body signal region. Each of the signal regions two-, three- and four-body are designed to target the event topologies coming from the signal models in Figure 6 . In each of the supersymmetric cases the number of bodies is defined as the number of particles emitted from the $\tilde{t}$ decay vertex, including the lightest supersymmetric particle or LSP $\left(\tilde{\chi}_{1}^{0}\right)$. In all cases energy escapes the detector, carried away by invisible particles. These invisible particles, the LSP in SUSY and $\chi$ in the DM model, are measured using the missing transverse momentum described earlier in this note (Section 3) and using the latest significance definition extra sensitivity can be gained to these new physics models.

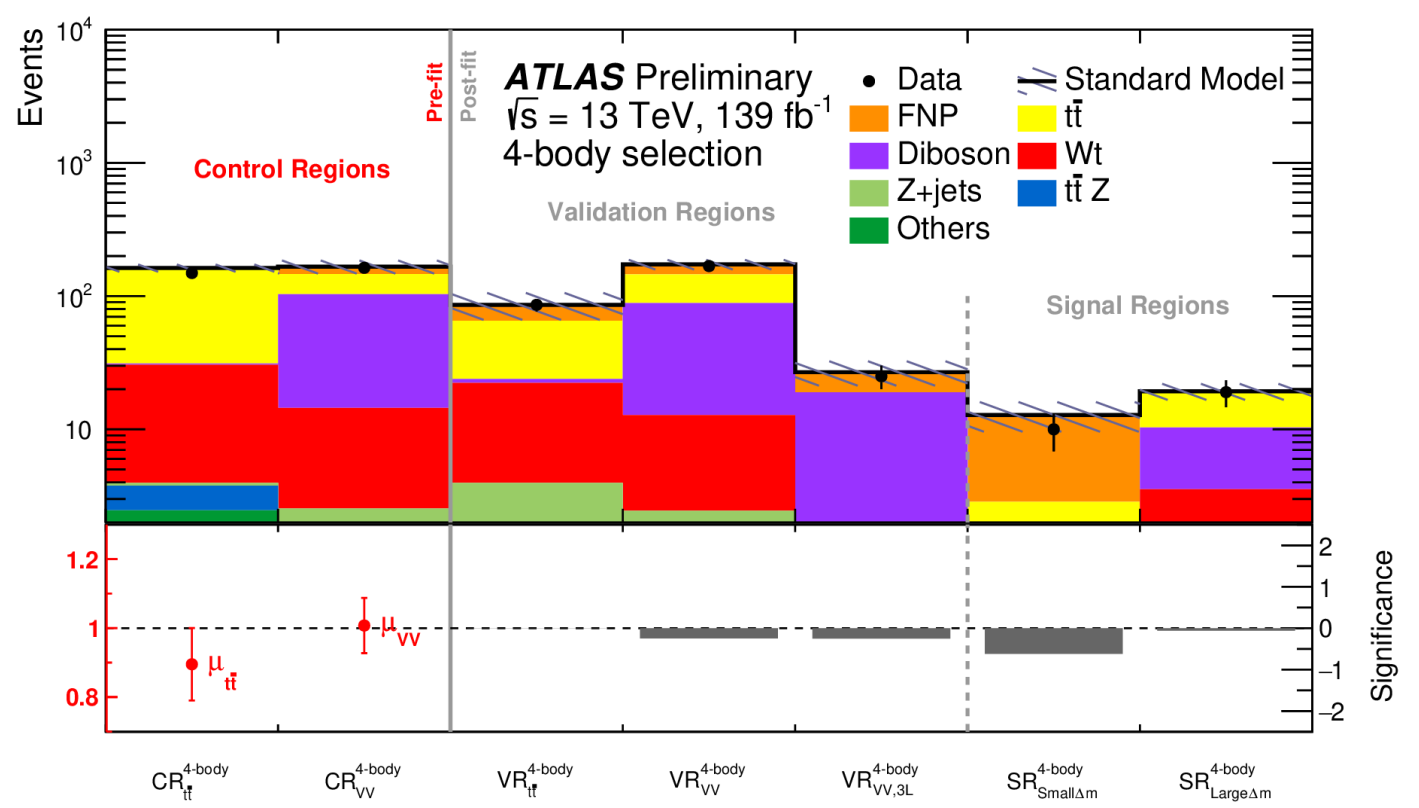

Figure 7: Four-body signal selection regions. The upper plot shows the observed and expected yields for the control, validation and signal regions from left to right. The two leftmost control region bins are prefit and the lower part of this plot shows the scale factors for these background contributions. The remaining lower panel plots show the significance for each region post-fit [10].

The analysis strategy aims to increase separation between SM background and potential signal. As a passing example the analysis has two variables for this purpose that are constructed form the $E_{\mathrm{T}}^{\text {miss }}$ and the $p_{\mathrm{T}}$ of the leading jet and lepton. These types of variables will be most improved by the use of the object-based missing transverse momentum and its significance. Figure 7 demonstrates the performance of the analysis for the four-body selection in the control, validation and signal regions. All of these show excellent agreement with the SM prediction and indicate that physics beyond the standard model has not been found on this occasion. Exclusion limits were then generated based on the findings and one of which is seen in Figure 8. There is a large improvement over the previous iteration of the search and this is beyond the simple addition of more statistics from the larger dataset. It is in fact due to the improved analysis techniques and in part due to the inclusion of the $E_{\mathrm{T}}^{\text {miss }}$ significance parameter. 


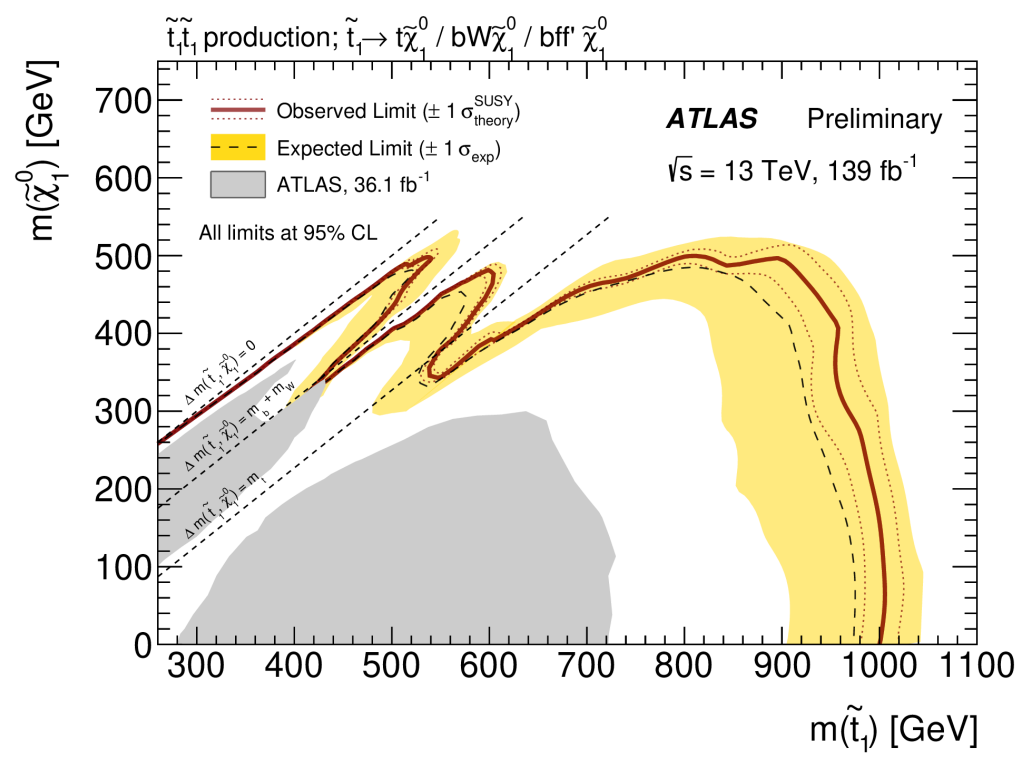

Figure 8: Contour of the exclusion limit with 95\% CL for the simplified SUSY model discussed above. This model assumes a $100 \%$ branching ratio for each of the pair produced $\tilde{t}$ to decay to a top quark and the LSP. The dashed lines set in the light band indicate the expected limit and the band represents the $\pm 1 \sigma$ error. The solid line shows the observed limits with the the dotted lines indicating the effect of varying the signal cross section by $\pm 1 \sigma$. The grey shaded areas highlight the old analysis limits when using $36 \mathrm{fb}^{-1}$ [10].

\section{SUSY Multi-jets 0 Lepton}

The second search to use the improved detector performance techniques is the multi-jet $0 \mathrm{~L}$. This analysis seeks complex final states that have many $(\geq 8)$ jets, no leptons and a moderate amount of missing transverse momentum. In a proton-proton collider there is a large predicted cross section for the production of gluinos $(\tilde{g})$. These massive particles would then decay through long decay chains that would be observed as many hadronic jets in ATLAS. There are some other subtleties to the analysis selections; $b$-tagging, to target SUSY models which preferentially decay to heavy flavour quarks and an event-level jet mass variable, to target scenarios in which the $\tilde{g}$ is particularly massive. Details on all of these features will not be covered here but can be found in the analysis paper [11].

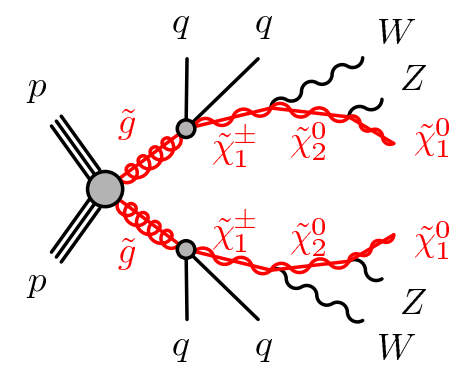

(a) Two-step

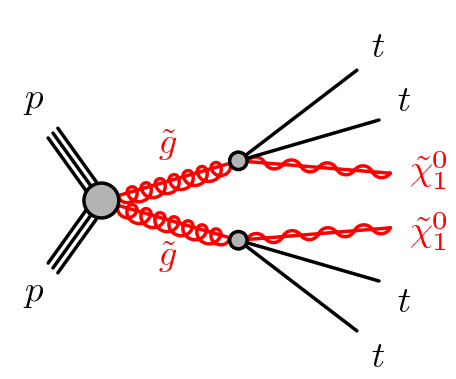

(b) Off-shell top squarks

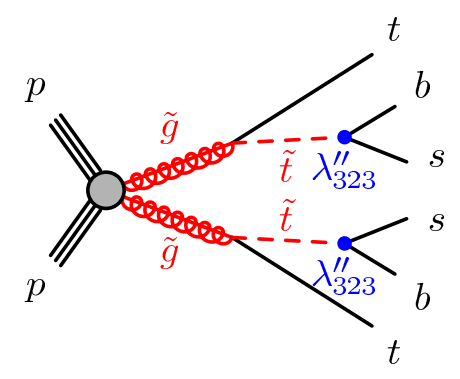

(c) RPV

Figure 9: The three signal models targeted by the search represented with pseudo-Feynman diagrams [11]. 
Figure 9 contains the three simplified models targeted by the search, these show that there will be a large number of jets in an event containing a pair of gluinos. The analysis is dependent on the efficacy of jet reconstruction, prompting the use of the latest jet reconstruction techniques, namely particle flow jets (Section 2). Another key component is the rejection of fake sources of $E_{\mathrm{T}}^{\text {miss }}$, that can come from jet mismeasurement and so is particularly important in such a jet rich environment. This search also employed the object-based $E_{\mathrm{T}}^{\text {miss }}$ significance alongside the PFlow jets and included the entire Run 2 dataset recorded by ATLAS with an integrated luminosity of $139 \mathrm{fb}^{-1}$.

The dominant background comes from QCD multi-jets originating from SM processes. Their contribution to the signal regions cannot be estimated using Monte Carlo and so a data-driven estimation is used. This method, known as the template method, is an ABCD method allowing the SM multi-jet contribution in a signal region to be constrained using three control regions. Figure 10 illustrates how the template method works. Two independent variables must be selected for an ABCD-type technique to function properly. In this case the number of jets in an event $N_{\text {jet }}$ and $\mathcal{S}\left(E_{\mathrm{T}}^{\text {miss }}\right)$ were chosen and manually shown to be independent of one another. Simple cuts in each dimension allow the definition of each of the regions; at a lower jet multiplicity than that of the signal region (SR) and with a large amount of missing transverse momentum significance $\left(\mathcal{S}\left(E_{\mathrm{T}}^{\mathrm{miss}}\right) \geq 5\right)$ the shape of of the $\mathcal{S}\left(E_{\mathrm{T}}^{\text {miss }}\right)$ distribution can be found.

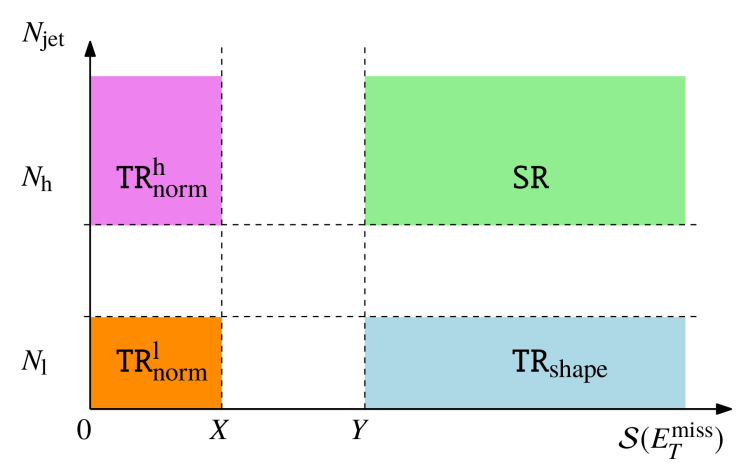

Figure 10: Schematic diagram of the regions used to estimate the number of background events in a signal region. 'SR' can represent any signal region and the template region (TR) types are regions of the phase space used to extract the background contribution to a given SR [11].

One of the key concepts in this technique is that the shape of the $\mathcal{S}\left(E_{\mathrm{T}}^{\text {miss }}\right)$ distribution is invariant under changes in $N_{\text {jet }}$ and only the normalisation of the distribution changes. This is intimately related to the initial $\mathrm{ABCD}$ method assumption that the variables are independent from each other. Once the shape has been found the distribution can be scaled to the SR using two normalisation regions; one at the same jet multiplicity as the template region but with $\mathcal{S}\left(E_{\mathrm{T}}^{\mathrm{miss}}\right) \leq 2$ and the second has the same $\mathcal{S}\left(E_{\mathrm{T}}^{\text {miss }}\right)$ condition as well as the same $N_{\text {jet }}$ as the SR. With these areas of phase space the background contribution to the SR can be estimated using the simple relation

$$
\hat{N}_{\mathrm{SR}}=\frac{N_{T R_{\text {norm }}^{h}}}{N_{T R_{\text {norm }}^{l}}} N_{T R_{\text {shape }}} .
$$

All regions in this analysis showed that observed data were in agreement with the standard model 
prediction. In the same fashion as the $\operatorname{tt}(2 \mathrm{~L})$ search discussed previously exclusion limits were found to have improved greatly. This improvement is well above what would have been expected from the simple addition of more data.

The data-driven background estimation as well as the large number of jets strongly motivated using improved jet reconstruction techniques in this search. The state of the art PFlow jets were employed over EMTopo for the main results, however, both jet types were used with the express intent of comparing their performance. When using PFlow jet reconstruction, the main background of the analysis was reduced by $25-30 \%$. There was also a reduction in the uncertainty related to the estimation of the QCD multi-jet background by 50\%. In short, not only was the principle background reduced by employing particle flow jets it was better constrained. This can be attributed to the improved pile up rejection achievable with PFlow meaning that the number of pileup jets incorrectly entering the analysis selections was reduced. All of these improvements combined result in a $30 \%$ or $1 \sigma$ increase in sensitivity.

\section{Conclusions and Outlook}

ATLAS recorded a phenomenal amount of data during Run 2 and it is extremely important to get as much as possible from this vast amount of information. Making the best measurements possible and maximising the possibility of discovering physics beyond the standard model are key goals for the collaboration. A great deal of effort has been invested in developing new techniques to improve the detector's performance, a few of which have been discussed in this note. Using particle flow jets has demonstrably improved the sensitivity of SUSY analyses and these are now the recommended jet type across the collaboration. Many searches for physics beyond the SM, including SUSY and DM, require the existence of a stable and invisible particle in the final state. Improved treatment and modelling of the missing transverse momentum increases the probability of searches reconstructing such a particle in an event. The latest formulation of missing transverse momentum significance allows for the rejection of fake sources of $E_{\mathrm{T}}^{\mathrm{miss}}$ and increases the likelihood that a measured amount of $E_{\mathrm{T}}^{\text {miss }}$ comes from a real invisible particle. All of these advances will form the basis of supersymmetry searches for Run 3 and allow for the development of superior techniques that will address the challenges of the HL-LHC and beyond. 


\section{References}

[1] The ATLAS Experiment at the CERN Large Hadron Collider, The ATLAS collaboration, 18th September 2008, Published in: JINST 3 (2008) S08003, https://inspirehep.net/ literature/796888

[2] Delivered Luminosity versus time for 2011-2018 (p-p data only), The ATLAS collaboration, https://twiki.cern.ch/twiki/bin/view/AtlasPublic/ LuminosityPublicResultsRun2\#Multiple_Year_Collision_Plots

[3] Jet energy scale and resolution measured in proton-proton collisions at $\sqrt{s}=13 \mathrm{TeV}$ with the ATLAS detector, The ATLAS collaboration, 6th July 2020, submitted to Eur. Phys. J. C, https://arxiv.org/abs/2007.02645 [hep-ex]

[4] Jet reconstruction and performance using particle flow with the ATLAS Detector, The ATLAS collaboration, 30th March 2017, final version published in Eur. Phys. J C, https: //arxiv . org/abs/1703.10485 [hep-ex]

[5] Topological cell clustering in the ATLAS calorimeters and its performance in LHC Run 1, The ATLAS collaboration, 9th March 2016, fEur. Phys. J. C 77 (2017) 490, https: //arxiv.org/abs/1603.02934 [hep-ex]

[6] $E_{\mathrm{T}}^{\text {miss }}$ performance in the ATLAS detector using 2015-2016 LHC p-p collisions, The ATLAS collaboration, 21st June 2018, ATLAS-CONF-2018-023, https: //cds . cern. ch/record/ 2625233

[7] Object-based missing transverse momentum significance in the ATLAS detector, The ATLAS collaboration, 14th July 2018, ATLAS-CONF-2018-038, https://atlas.web.cern.ch/ Atlas/GROUPS/PHYSICS/CONFNOTES/ATLAS-CONF-2018-038/

[8] Missing transverse momentum performance using the full run $2 \mathrm{pp}$ data at $13 \mathrm{TeV}$, The ATLAS collaboration, 19th February 2019, JETM-2019-03, https://atlas.web.cern . ch/Atlas/GROUPS/PHYSICS/PLOTS/JETM-2019-03/

[9] TST systematics using 2015-2018 data, The ATLAS collaboration, 21 July 2020, JETM-202002, https://atlas. web. cern. ch/Atlas/GROUPS/PHYSICS/PLOTS/JETM-2020-02/

[10] Search for new phenomena in events with two opposite-charge leptons, jets and missing transverse momentum in pp collisions at $\sqrt{s}=13 \mathrm{TeV}$ with the ATLAS detector, The ATLAS collaboration, 27th July 2020, ATLAS-CONF-2020-046, https://atlas . web.cern.ch/ Atlas/GROUPS/PHYSICS/CONFNOTES/ATLAS-CONF-2020-046/

[11] Search for new phenomena in final states with large jet multiplicities and missing transverse momentum using $\sqrt{s}=13 \mathrm{TeV}$ proton-proton collisions recorded by ATLAS in Run 2 of the LHC, The ATLAS collaboration, 20th February 2020, CERN-EP-2020-123, https: //cds.cern.ch/record/2728000 
[12] Supersymmetric Searches Public Event Displays $[13$ and 16 Jets], the ATLAS collaboration, https://twiki.cern.ch/twiki/bin/view/AtlasPublic/ EventDisplayRun2Physics\#SUSY 